\title{
Health-related quality of life in persons with West Nile virus infection: a longitudinal cohort study
}

\author{
Man Wah Yeung ${ }^{1}$, George Tomlinson ${ }^{2,3,4}$, Mark Loeb ${ }^{5}$ and Beate Sander ${ }^{1,2,6,7^{*}}$
}

\begin{abstract}
Background: West Nile virus (WNV) infections are predominantly asymptomatic, although almost 1\% become neuroinvasive and debilitating. We describe the impact of neuroinvasive and non-neuroinvasive disease on patient health-related quality of life (HRQoL).

Methods: Short Form 36 questionnaire data came from a Canadian WNV cohort (Loeb 2008) of 154 patients followed for up to three years. We generated health utilities using the SF-6D. We calculated mean utility scores throughout follow-up and examined predictors using a linear mixed-effects model. We summarized HRQoL postacute infection as: (i) long-term utility (mean of scores one year onward); (ii) area under the curve (AUC) one year onward. We examined predictors using beta regression. We used multiple imputation for sensitivity analysis.
\end{abstract}

Results: Mean utility scores improved from $0.59(95 \% \mathrm{Cl}: 0.38,0.93)$ at baseline to $0.77(0.53,1)$ at six months, before plateauing for the remaining two years. Mean long-term utility was $0.81(0.78,0.85)$ and mean AUC was $0.80(0.76$, 0.84). Patients with neuroinvasive disease had consistently worse scores than their non-neuroinvasive counterparts, with the gap nearly closed after six months. After adjusting for confounding, neuroinvasive disease was not a significant predictor of HRQoL either throughout follow-up or post-acute infection. Rather, number of comorbidities and baseline utility scores were. Sensitivity analysis showed similar findings.

Conclusions: Patients with WNV infection reported low HRQoL during acute illness, but improved rapidly by six months, regardless of neuroinvasive disease status. This is the first study reporting health utilities for WNV infection.

Keywords: West Nile virus, Health-related quality of life, Short-form 36, Short-form 6D, Preferences

\section{Background}

West Nile virus (WNV) has firmly established itself in North America since 1999. Symptomatic infections are mainly febrile (non-neuroinvasive), but almost $1 \%$ of infections become neuroinvasive [1], with debilitating physical and cognitive effects over the short and potentially long term.

There is a need to understand the impact of WNV infection from the patient perspective. Measures of health-related quality of life (HRQoL) capture the social, emotional and physical domains of the patient. HRQoL can be expressed as utility scores, which capture patient preferences for different health states and are central to calculating cost-utility in economic evaluations [2]. In WNV infection, few studies have examined physical and mental impairments and none have accounted for patient preferences $[3,4]$. As such, we describe HRQoL as utility scores in patients with WNV infection from a cohort study, comparing those with neuroinvasive and non-neuroinvasive diseases. Further, we examined sociodemographic and clinical characteristics associated with HRQoL.
* Correspondence: beate.sander@theta.utoronto.ca

${ }^{1}$ Public Health Ontario, 480 University Avenue, Suite 300, Toronto, ON M5G

$1 \mathrm{~V} 2$, Canada

${ }^{2}$ Institute of Health Policy, Management and Evaluation, University of

Toronto, 155 College St, Toronto, ON M5T 3M6, Canada

Full list of author information is available at the end of the article

\section{Methods \\ Ethics}

The present work is part of a larger study, Cost-effectiveness of West Nile virus intervention strategies. A 
computer simulation (Canadian Institutes of Health Research grant: MOP133571), approved by the Ontario Agency for Health Protection and Promotion institutional review board. Data were obtained from a previous study from McMaster University (MOP-69010), which had received ethics approval from McMaster University, University of Manitoba, University of Saskatchewan, and University of Alberta [5].

\section{Data source}

Data were obtained from an existing cohort study of 156 Canadian adults with confirmed WNV infection [5]. From provincial laboratory testing, patients with positive results were recruited by physicians and confirmed to have WNV through hospital, clinic, and laboratory records. Patients were predominantly from Ontario (65\%), as well as Manitoba, Saskatchewan, and Alberta. Data were collected on sociodemographics, and comorbid diagnoses including cardiac disease, peripheral vascular disease, chronic obstructive, pulmonary disease, diabetes, renal failure, peptic ulcer disease, cancer, and rheumatologic disease. The study aimed to recruit patients within four weeks of symptom onset and interviewed patients during home visits or in ambulatory care over their follow-up of up to three years. The study measured patient HRQoL using the Medical Outcomes Survey Short Form 36 (SF-36) questionnaire, which consists of 36 questions that can be summed into eight subscales (physical functioning, social role functioning, bodily pain, general health perceptions, role limitations due to physical health, role limitation due to emotional problems, mental health, vitality) [6]. Each subscale has a score from 0 (maximum disability) to 100 (no disability). The eight subscales can be further aggregated into the Physical Component Summary and Mental Component Summary scores, which also range from 0 (maximum disability) to 100 (no disability).

\section{Measurements \\ Exposure}

The diagnostic criteria for neuroinvasive diseases (encephalitis, meningitis, meningoencephalitis, and acute flaccid paralysis) are described elsewhere [5]. Patients who did not meet these criteria were classified having non-neuroinvasive disease.

\section{Outcomes}

The SF-36 questionnaire used is not based on preferences and therefore does not directly provide utility scores. To generate utility scores, we converted responses using the Medical Outcomes Study Short Form 6D classification (SF-6D) obtained from the University of Sheffield [7]. The algorithm combines selected SF-36 items into six health subscales (physical functioning, social functioning, bodily pain, role participation, mental health, vitality). The SF-6D uses preference weights from the United Kingdom general population based on the standard gamble technique, where individuals choose between a hypothetical outcome to occur with certainty (i.e., suboptimal health) or a gamble (i.e., probability of either perfect health or sudden death) [8]. Parametric and non-parametric models have been developed to predict all 18,000 health states described by the SF-6D [7, 9]. Utility scores range from 0 (equivalent to being dead) to 1 (equivalent to perfect health).

\section{Statistical analysis}

We performed analyses in SAS version 9.3 (SAS Institute Inc., Cary, NC, USA) and R 3.3.2 [10]. Continuous variables were compared between those with and without neuroinvasive disease using Student's t-test. Categorical variables were compared using the Chi-squared test or Fisher's exact test. In the main analyses, we used all available data but excluded patients who did not complete the SF-36 questionnaire at any follow-up visit.

\section{Mean utilities by time}

We calculated the mean utility scores at different time points over their follow-up visits in strata formed by sex and baseline values of neuroinvasive disease status, age, and comorbidities. To calculate the $95 \%$ confidence intervals $(\mathrm{CI})$, we used the 2.5th and 97.5th percentiles from 2000 bootstrap samples.

\section{Predictors of HRQoL over entire follow-up}

We modelled both logit-transformed utility scores (to account for the data being bounded by the open interval $(0,1))$ and untransformed utility scores (for easier interpretability). To examine the association of patient characteristics with utility scores over time, we fitted linear mixed-effects models to all observations after baseline. All models had fixed effects for time (elapsed since baseline) and random effects for the intercept and time. Additional fixed effects considered were neuroinvasive disease status, age, sex, number of comorbid conditions, baseline utility scores, and the interaction of neuroinvasive disease with time. Each covariate was added alone to the base model and two additional models were fitted: one with all covariates, and one with all covariates except the interaction. Correlation between residuals was addressed through use of either an autoregressive (AR1) structure or an exponential power function based on time between measurements. As a further check on the results of the logit-transformed model, a beta-regression based random effects model was also fitted. 


\section{Response feature analysis}

We collapsed a patient's repeated HRQoL measures into single indices known as response features [11], focusing on two longer-term intervals: (i) from six months onward, or (ii) from one year onward The first response feature was the mean score over observations in the interval. The second response feature was the area under the curve (AUC), calculated using the trapezoid rule and then divided by the relevant follow-up time $[11,12]$. This assumes that missing values fell linearly between existing observations. AUC can be interpreted as a time-weighted average HRQoL. These response features represented HRQoL post-acute infection, with larger values signifying better HRQoL.

\section{Predictors of HRQoL response features}

We used beta regression to examine the association between neuroinvasive disease and HRQoL post-acute infection. Beta regression accounts for the HRQoL data being bounded by the open unit interval $(0,1)$. HRQoL scores of one were adjusted to slightly smaller values (0.995) [13]. Specifically, we regressed mean long-term utility scores or AUC scores on neuroinvasive disease, adjusting for age (centered at 50 years old), sex, number of comorbid conditions, and baseline utility scores (centered at 0.5 ). Baseline utilities act as a proxy for other unmeasured confounders. We used the $\mathrm{R}$ betareg package [14] with a logit link, with and without parameterization of the precision as a function of the predictors [13].

\section{Sensitivity analysis}

Utility score outcomes were missing when specific SF-36 responses were not completed or when patients missed study visits. Given attrition of the cohort, we checked for patterns of missing data across visits and determined whether having missing data at one visit was related to utility scores from the previous visit. We used multiple imputation by predictive mean matching, running 50 sets of imputation and taking the 100th sequential sample as the imputed dataset. We recalculated the response features on each imputed dataset, reran the beta regression model on each dataset and combined the estimated coefficients using Rubin's rules [15].

\section{Role of the funding source}

The funder had no role in the study design, analysis, decision to publish, or manuscript preparation. The corresponding author had full access to the data and had final responsibility for the decision to publish.

\section{Results}

The cohort enrolled 156 patients. Two patients who did not complete the SF-36 questionnaire at any visit were excluded for a final sample of 154 patients (Fig. 1) [5]. Patients were middle-aged with few comorbid conditions (mean: 0.9, standard deviation: 0.1) (Table 1). Sixty-two patients $(40 \%)$ had neuroinvasive

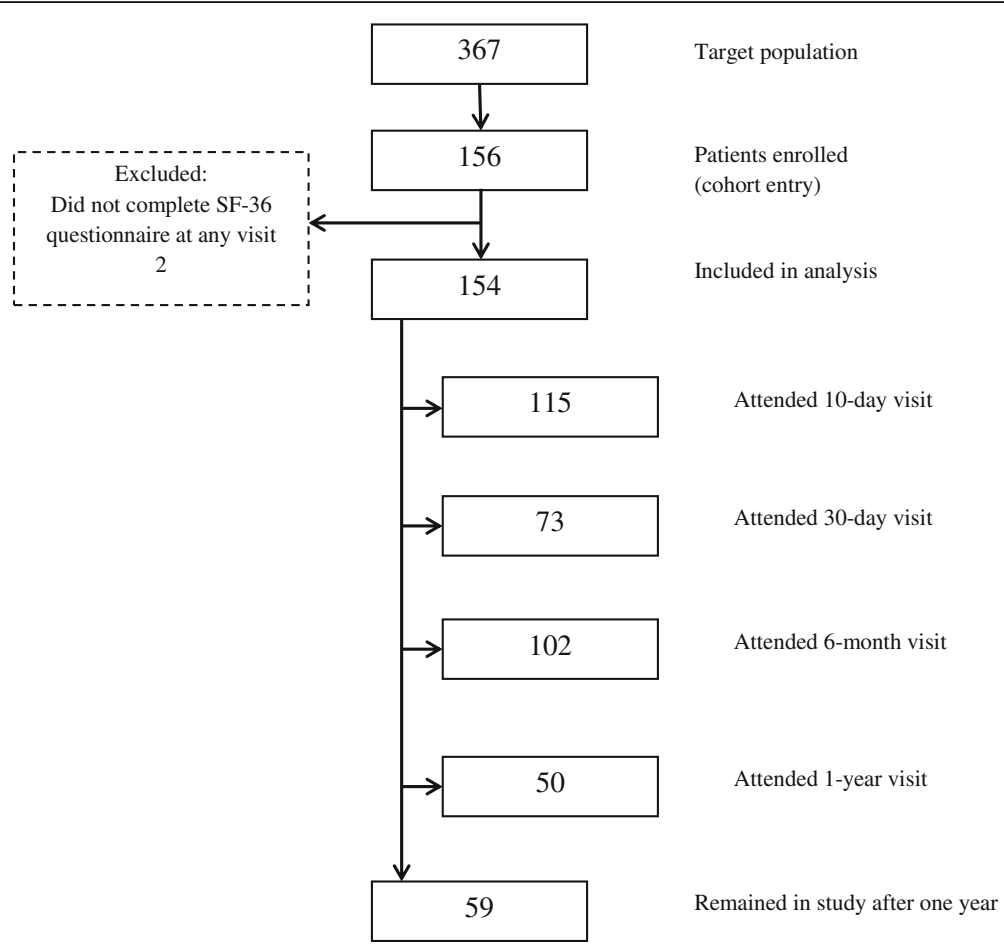

Fig. 1 Study flow 
Table 1 Patient characteristics at cohort entry

\begin{tabular}{|c|c|c|c|}
\hline & \multirow{2}{*}{$\begin{array}{l}\text { Total } \\
(n=154)\end{array}$} & \multicolumn{2}{|c|}{ Disease classification } \\
\hline & & $\begin{array}{l}\text { Neuroinvasive } \\
(n=62)\end{array}$ & $\begin{array}{l}\text { Nonneuroinvasive } \\
(n=92)\end{array}$ \\
\hline Mean age $(s d)$, years ${ }^{a}$ & $52(13)$ & $55(15)$ & $50(12)$ \\
\hline Female (\%) & $75(49)$ & $26(42)$ & $49(53)$ \\
\hline Mean follow up (sd), months ${ }^{a}$ & $11(9)$ & $11(6)$ & $9(5)$ \\
\hline Lost to follow-up at one year (\%) & $95(62)$ & $32(52)$ & $63(68)$ \\
\hline \multicolumn{4}{|l|}{ Comorbid conditions (\%): } \\
\hline Asthma & $14(9)$ & $4(6)$ & $10(11)$ \\
\hline $\mathrm{COPD}^{\mathrm{a}}$ & $4(3)$ & $4(6)$ & $0(0)$ \\
\hline Cancer & $17(11)$ & $5(8)$ & $12(13)$ \\
\hline Cardiac disease & $21(14)$ & $12(19)$ & $9(10)$ \\
\hline Cerebrovascular disease & $3(2)$ & $2(3)$ & $1(1)$ \\
\hline Diabetes $^{\mathrm{a}}$ & $11(7)$ & $9(15)$ & $2(2)$ \\
\hline Liver disease $^{a}$ & $6(4)$ & $5(8)$ & $1(1)$ \\
\hline Lung disease & $16(10)$ & $9(15)$ & $7(8)$ \\
\hline Renal disease & $7(5)$ & $4(6)$ & $3(3)$ \\
\hline Transplant & $5(3)$ & $3(5)$ & $2(2)$ \\
\hline Mean number of comorbid conditions (sd) ${ }^{a}$ & $0.9(0.10)$ & $1.2(0.2)$ & $0.7(0.1)$ \\
\hline Prior hospitalizations (\%) & $118(77)$ & $51(82)$ & $67(73)$ \\
\hline \multicolumn{4}{|l|}{ Disease classification (\%) } \\
\hline Neuroinvasive & $62(40)$ & - & - \\
\hline Encephalitis & $21(14)$ & - & - \\
\hline Meningitis & $3(2)$ & - & - \\
\hline Acute flaccid paralysis & $6(4)$ & - & - \\
\hline Meningo-encephalitis & $32(21)$ & - & - \\
\hline Nonneuroinvasive & $92(60)$ & - & - \\
\hline
\end{tabular}

Abbreviations: sd, standard deviation; COPD, chronic obstructive pulmonary disease

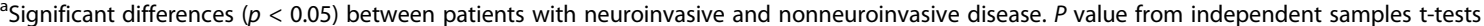

disease, and the remaining had non-neuroinvasive disease. There were 108 patients $(70 \%)$ followed past six months, and 59 (38\%) past one year. There were no discernible patterns in the missing HRQoL outcome across visits.

\section{Statistical analysis}

Mean utilities by time

At baseline, the entire cohort had a mean utility score of 0.59 (95\% CI: 0.57, 0.62), which improved rapidly over the subsequent months (Table 2). Qualitatively, the

Table 2 Mean utilities by time: Utility scores ${ }^{\mathrm{a}}$ (95\% confidence interval) at select study visits

\begin{tabular}{llllllll}
\hline & Cohort entry & 10-day visit & 30-day visit & 6-month visit & 12-month visit & 24-month visit & 30-month visit \\
\hline Total cohort & 0.59 & 0.67 & 0.72 & 0.77 & 0.80 & 0.76 & 0.77 \\
& $(0.57,0.62)$ & $(0.64,0.69)$ & $(0.69,0.75)$ & $(0.74,0.80)$ & $(0.76,0.84)$ & $(0.66,0.84)$ & $(0.69,0.84)$ \\
& $n=146$ & $n=106$ & $n=68$ & $n=95$ & $n=49$ & $n=12$ & $n=10$ \\
Neuro-invasive & 0.54 & 0.60 & 0.67 & 0.74 & 0.81 & 0.75 & 0.77 \\
& $(0.51,0.57)$ & $(0.57,0.64)$ & $(0.63,0.72)$ & $(0.69,0.78)$ & $(0.76,0.86)$ & $(0.65,0.85)$ & $(0.68,0.86)$ \\
Non-neuro-invasive & $n=58$ & $n=38$ & $n=21$ & $n=39$ & $n=26$ & $n=8$ & $n=6$ \\
& 0.63 & 0.70 & 0.74 & 0.79 & 0.80 & 0.77 & 0.76 \\
& $(0.60,0.66)$ & $(0.67,0.73)$ & $(0.70,0.78)$ & $(0.76,0.83)$ & $(0.73,0.85)$ & $(0.57,0.89)$ & $(0.61,0.86)$ \\
& $n=88$ & $n=68$ & $n=47$ & $n=56$ & $n=23$ & $n=4$ & $n=4$ \\
\hline
\end{tabular}

a Utility scores range on a scale from 0 (equivalent to being dead) to 1 (equivalent to perfect health). Larger values indicate better health-related quality of life. Scores are derived from the Medical Outcomes Study Short-Form-6D health state classification 
utility scores plateaued at six months at 0.77 (95\% CI: $0.74,0.80)$, and remained fairly steady over the remaining two years. The trend was similar in the subset of patients with at least one year of follow-up, and in patients stratified by age, sex, and comorbidity (Fig. 2). Specifically for patients with neuroinvasive disease, the mean utility score at baseline $(0.54 ; 95 \%$ CI: $0.51,0.57)$ was lower than that of their non-neuroinvasive counterparts $(0.63$; 95\% CI: $0.60,0.66)$. By one year however,

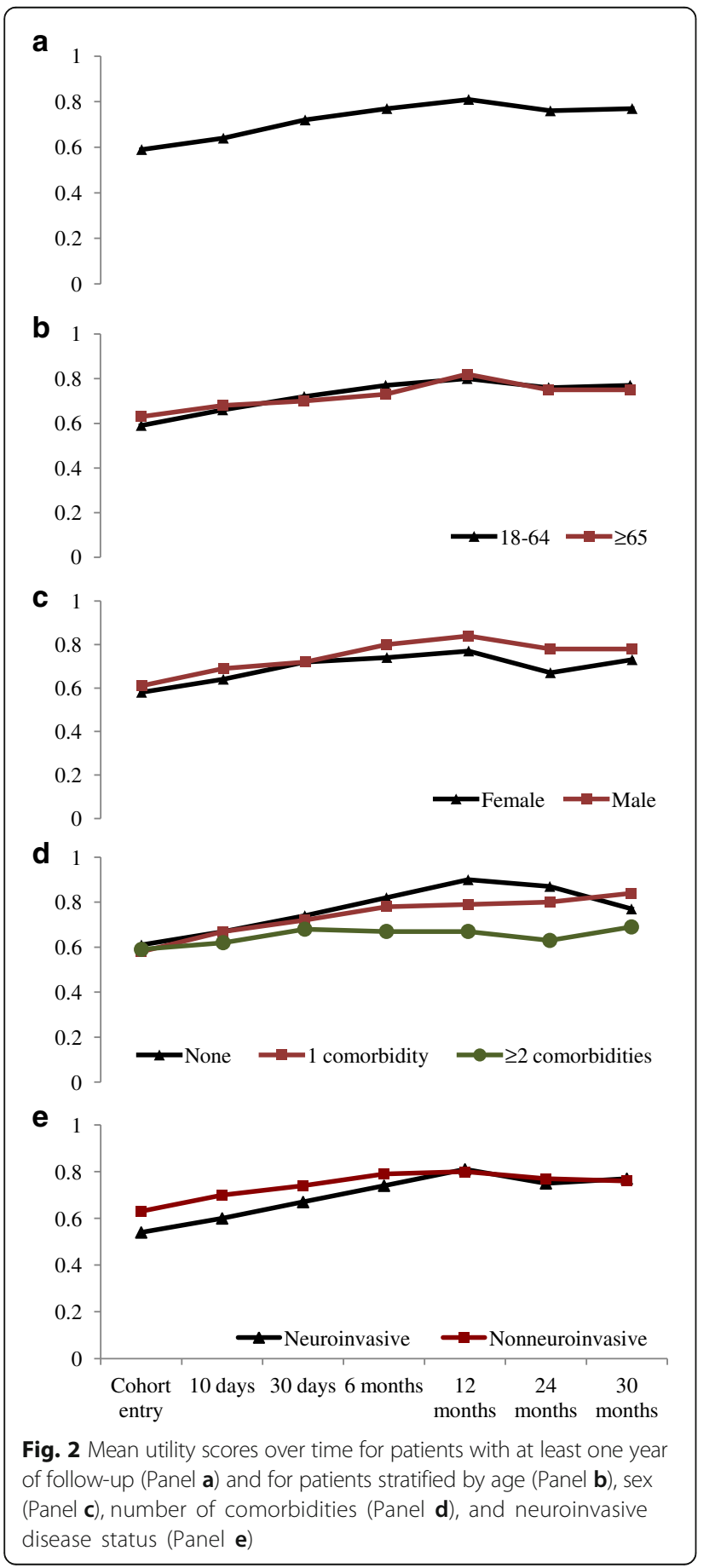

their HRQoL improved to the levels of those with nonneuroinvasive disease $(0.81$ (95\% CI: $0.76,0.86)$ versus 0.80 (95\% CI: 0.73, 0.85)).

\section{Predictors of HRQoL over entire follow-up}

Table 3 shows the linear mixed-effects model results for logit-transformed utility scores (Additional File 1 for untransformed scores). Results of the beta-regression based random effects model were similar (not shown). Neuroinvasive disease, gender, baseline utility scores, and number of comorbidities were significant predictors of HRQoL in the univariate analysis. However in the final model including all covariates, neuroinvasive disease status was no longer significant. It only appeared significant when baseline scores were excluded from the model.

In the final model, the intercept represents the mean logit HRQoL score when all covariates, and random effects are zero-that is, the logit HRQoL of a 50 year old female patient with non-neuroinvasive disease, no comorbidities, and a baseline utility score of 0.50 . The intercept of 1.00 would be interpreted as a fitted utility score of $\frac{e^{1.00}}{1+e^{1.00}}=0.73$. Different patient characteristics would vary one's absolute HRQoL. For instance, a 60 year old male with non-neuroinvasive disease, one comorbid condition, and a baseline utility score of 0.60 would have the following logit score 1.00 (intercept) +0 (non-neuroinvasive) - 0.01 (ten years greater than 50 years old) +0.25 (male) -0.23 (one comorbidity) $+2.17 * 0.10$ (baseline utility was 0.10 greater than 0.50$)=1.247$, corresponding to a fitted utility score of 0.78 .

\section{Response feature analysis}

Patients in the total cohort had similar AUC (Table 4) and mean long-term utilities (Additional File 2). Utilities were also similar regardless of the time period captured. Time-weighted-AUC scores were 0.78 (95\% CI: 0.75, 0.80 ) from six months onward, and 0.81 (95\% CI: 0.78 , 0.84) from one year onward. Patients with neuroinvasive disease had consistently lower HRQoL scores than those with non-neuroinvasive disease.

\section{Predictors of HRQoL response features}

Table 5 shows the beta regression results for AUC, with and without multiple imputation. Number of comorbidities, and baseline utility scores were statistically significant for AUC from six months onward. However, only comorbidities were significant from one year onward. Effect sizes were reduced with imputation. Figure 3 shows the effect of each additional comorbid condition in a patient. The effect of comorbid conditions is as large as moving from the 5th to 95th percentile of observed baseline utilities. Neuroinvasive disease status did not 
Table 3 Linear mixed-effects model for predictors of health-related quality of life over entire follow-up

\begin{tabular}{|c|c|c|c|}
\hline & \multicolumn{3}{|c|}{ Fixed effects (95\% confidence interval) } \\
\hline & Time only model & $\begin{array}{l}\text { One additional } \\
\text { covariate in model }\end{array}$ & All covariates in model \\
\hline Intercept & $\begin{array}{l}1.07 \\
(0.95,1.20)\end{array}$ & (varies) & $\begin{array}{l}1.00 \\
(0.80,1.20)\end{array}$ \\
\hline Neuroinvasive disease & - & $\begin{array}{l}-0.36 \\
(-0.60,-0.11)\end{array}$ & $\begin{array}{l}-0.09 \\
(-0.32,0.13)\end{array}$ \\
\hline Age (per 10 years), centered at 50 years & - & $\begin{array}{l}-0.05 \\
(-0.14,0.04)\end{array}$ & $\begin{array}{l}-0.01 \\
(-0.09,0.07)\end{array}$ \\
\hline Male & - & $\begin{array}{l}0.25 \\
(0.0,0.48)\end{array}$ & $\begin{array}{l}0.25 \\
(0.04,0.45)\end{array}$ \\
\hline Number of comorbid conditions & - & $\begin{array}{l}-0.24 \\
(-0.34,-0.14)\end{array}$ & $\begin{array}{l}-0.23 \\
(-0.32,-0.13)\end{array}$ \\
\hline Baseline utility score, centered at 0.50 & - & $\begin{array}{l}2.41 \\
(1.6,03.21)\end{array}$ & $\begin{array}{l}2.17 \\
(1.38,2.95)\end{array}$ \\
\hline Time elapsed since baseline (years) & $\begin{array}{l}0.73 \\
(0.56,0.90)\end{array}$ & (varies) & $\begin{array}{l}0.59 \\
(0.38,0.80)\end{array}$ \\
\hline $\begin{array}{l}\text { Interaction between neuroinvasive } \\
\text { disease and years elapsed }\end{array}$ & - & - & $\begin{array}{l}0.02 \\
(-0.28,0.31)\end{array}$ \\
\hline
\end{tabular}

Results are measured as logit-transformed utility scores. Utility scores range on a scale from 0 (equivalent to death) to 1 (equivalent to perfect health) Significant results are bolded

The coefficients represent the change in the mean logit of utility scores when the predictor increases by one unit and the remaining covariates are held constant

have as much effect on predicted AUC as the number of comorbidities (Additional File 3).

Predicted mean AUC from the regression (with and without imputation) were similar to observed scores from Table 4. For example, a 60 year old male with neuroinvasive disease, one comorbid condition, and a baseline utility score of 0.60 would have a predicted AUC past six months equal to 0.81 . Patients with neuroinvasive disease had a mean AUC of 0.80 observed.

\section{Discussion}

We described HRQoL longitudinally as utility scores in a Canada-wide cohort of patients with WNV. At

Table 4 Response feature analysis: Utility scores ${ }^{\mathrm{a}}(95 \%$ confidence interval) summarized into area under the curve

\begin{tabular}{lll}
\hline & Area under the curve & \\
\hline & Six months onward & One year onward \\
Total cohort & 0.78 & 0.81 \\
& $(0.76,0.81)$ & $(0.77,0.84)$ \\
& $n=108$ & $n=59$ \\
Neuroinvasive & 0.75 & 0.80 \\
& $(0.71,0.79)$ & $(0.75,0.85)$ \\
Nonneuroinvasive & $n=45$ & $n=30$ \\
& 0.80 & 0.82 \\
& $(0.77,0.83)$ & $(0.76,0.86)$ \\
& $n=56$ & $n=29$ \\
\end{tabular}

a Utility scores range on a scale from 0 (equivalent to being dead) to 1 (equivalent to perfect health). Larger values indicate better health-related quality of life. Scores are derived from the Medical Outcomes Study Short-Form-6D health state classification

Note: One patient missing utility scores at all visits

${ }^{\mathrm{b}}$ Area under the curve is the integral of the utility-time curve. Areas are timeweighted (Total area after one year $\div$ follow-up time) baseline, patients had an impaired HRQoL (mean: 0.59 (95\% CI: $0.57,0.62)$ ) that improved rapidly, and plateaued at about six months of follow-up (mean: 0.77 (95\% CI: 0.74, 0.80)). Throughout follow-up, patients with neuroinvasive disease fared consistently worse than their non-neuroinvasive counterparts, although the gap closed by one year. After adjusting for confounding, neuroinvasive disease was no longer a significant predictor of HRQoL. Both the mixed-effects model on HRQoL over time, and the beta regression model on HRQoL post-acute infection showed comorbidities, and baseline HRQoL were significant predictors. This finding is similar to a New York City study that noted better health one year after WNV onset in patients with no comorbidities versus patients with hypertension or diabetes [16]. It reported greater recovery in physical, cognitive, and functional health domains among patients with no comorbidities (risk ratio:2.1 (95\% CI: 0.80-5.6)) [16].

Patients after acute infection did not recover to "perfect health", which would have been indicated by a utility score of 1 . It is not known if patients recovered to levels prior to WNV infection since HRQoL data were collected from cohort enrollment onward. Reference utility scores, however, exist among Canadian community-dwellers [17]. The general population without chronic conditions had qualitatively better utility scores than our sample throughout follow-up (mean: $0.93 \pm 0.079$ ). The general population in an age group similar to our sample (50 to 59 year olds) had a mean score of $0.92 \pm 0.070$. These scores were derived from the Health Utilities Index (HUI)-Mark III [18], which uses a time trade-off technique unlike the SF-6D 
Table 5 Beta regression model for predictors of health-related quality of life summarized into area under the curve ${ }^{a}$

\begin{tabular}{|c|c|c|c|c|}
\hline & \multicolumn{4}{|c|}{ Coefficients $^{\mathrm{b}}$ (95\% confidence interval) } \\
\hline & \multicolumn{2}{|l|}{ No imputation } & \multicolumn{2}{|l|}{ Multiple imputation } \\
\hline & Six months onward & One year onward & Six months onward & One year onward \\
\hline Intercept & $1.26(0.98,1.54)$ & $1.91(1.66,2.16)$ & $1.31(1.12,1.49)$ & $1.39(1.19,1.58)$ \\
\hline Neuroinvasive disease & $0.03(-0.27,0.34)$ & $0.04(-0.23,0.30)$ & $0.02(-0.17,0.22)$ & $0.01(-0.18,0.19)$ \\
\hline Age per 10 years, centered at 50 years & $-0.08(-0.19,0.03)$ & $-0.08(-0.18,0.014)$ & $-0.03(-0.10,0.04)$ & $-0.02(-0.09,0.05)$ \\
\hline Male & $0.26(-0.02,0.53)$ & $0.02(-0.29,0.33)$ & $0.15(-0.03,0.33)$ & $0.10(-0.08,0.27)$ \\
\hline Number of comorbid conditions & $-0.25(-0.35,-0.14)$ & $-0.46(-0.62,-0.30)$ & $-0.16(-0.24,-0.09)$ & $-0.13(-0.21,-0.05)$ \\
\hline Baseline utility (centred) & $1.70(0.74,2.67)$ & $0.58(-0.52,1.68)$ & $0.84(0.06,1.62)$ & $0.29(-0.61,1.18)$ \\
\hline
\end{tabular}

Statistically significant results are in bold

${ }^{a}$ Area under the curve is the integral of the utility-time curve after one year. Areas are time-weighted (Total area after one year $\div$ follow-up time)

${ }^{b}$ Coefficients and patient characteristics can be substituted into the following equation to calculate the area under the curve:

$\operatorname{Logit}($ Mean $($ Utility score $))=\beta_{0}+\beta_{1}($ Neuroinvasive disease $)+\beta_{2}\left(\frac{\text { Age-50 years old }}{10}\right)+\beta_{3}($ male $)+\beta_{4}$ (No.comorbid conditions $)+\beta_{5}($ Baseline utility score-0.50)

For example, the regression equation for area under the curve past six months in a 60 year old male with neuroinvasive disease, one comorbid condition and a baseline utility score of 0.60 would be: 1.31 (intercept) +0.02 (neuroinvasive) -0.03 (ten years greater than 50 years old) +0.15 (male) -0.16 (one comorbid condition) $+0.84 * 0.10$ (baseline utility score was 0.10 greater than 0.50$)=1.434$. Taking the inverse logit, this particular patient would have a predicted longterm utility score of: $\frac{e^{1.434}}{1+e^{1.434}}=0.81$

which uses standard gamble. HUI-Mark III also differs in the health domains assessed, notably in vision, hearing, speech, and dexterity.

To our knowledge, this is the first study to report utility scores for the WNV patient population. Such weights are essential in cost-utility analyses for calculating quality-adjusted life-years. Quality-adjusted life-years are a function of health utilities over time. The few existing WNV economic evaluations used utility scores taken from other diseases: unspecified neurologic conditions (0.75) [19]; herpes simplex in the central nervous system, and Haemophilus influenzae vaccination sequelae (values not reported) [20]. Keeping in mind future economic evaluations, we present health utilities at different time points, stratified by patient characteristics including neuroinvasive disease, age, sex, and comorbidities, and present summary scores of HRQoL post-acute infection.

The SF-6D instrument incorporates general population weights from the United Kingdom [7], which we applied to patients in Canada where general population weights are unavailable. Valuation of health may differ between the two countries. Other studies examining psychological or physical quality of life domains have used instruments including the Depression Anxiety Stress Scale, Beck Depression Inventory II, Fatigue Severity Scale, Modified Fatigue Impact Scale, Barthel Index of Activities of Daily Living, and Modified Rankin Scale [3, 21-23]. Only Hasbun et al., and Carson et al. used instruments that could generate utilities-namely, SF-36, and its abbreviated version, Short Form 12 (SF-12) [22, 23]. Both the

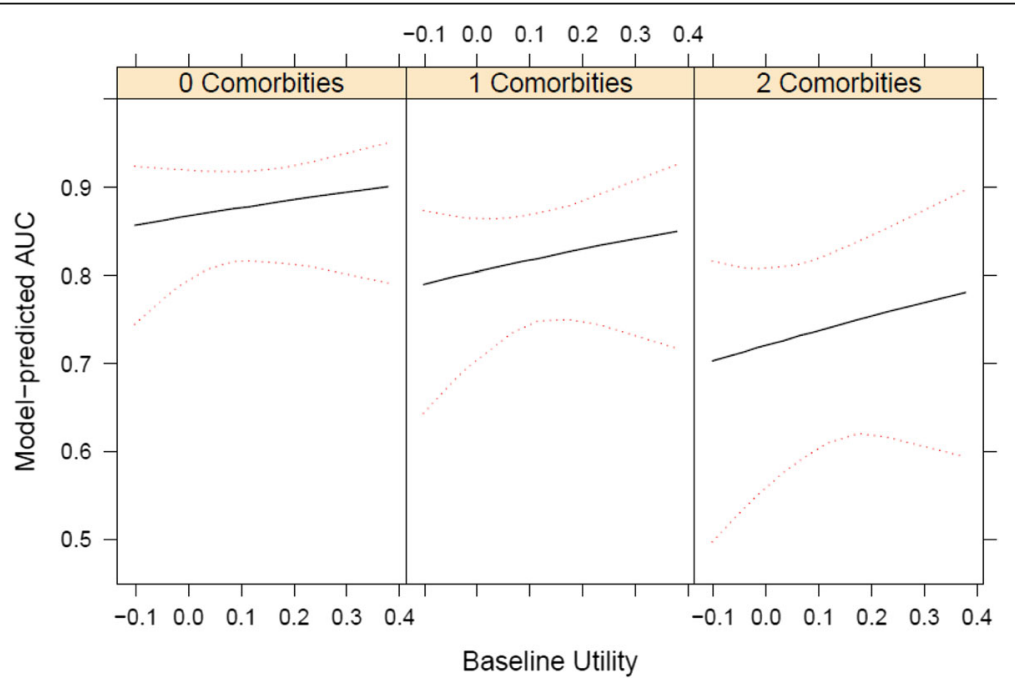

Fig. 3 Predicted values from best beta-regression model for area under the curve (AUC) past one year with bootstrap confidence intervals 
SF-36 and SF-12 are generic rather than disease-specific HRQoL instruments, meaning they can be applied to, and compared across different medical conditions, and patient populations. However, generic instruments may not capture neurological attributes particular to WNV. For instance, fever, stiff neck, headache, weak muscles, gastrointestinal symptoms, disorientation, tremors, convulsions, and paralysis are manifestations of neuroinvasive disease [24]. At this point, WNV-specific HRQoL instruments have not been developed. Neurology-specific instruments may be considered for use in conjunction with general instruments, just as arthritis-specific instruments have been accepted for use in another arbovirus, chikungunya, specifically for its rheumatism sequelae [25].

The two studies that used the Short Form questionnaires may not be comparable to our present work. Hasbun et al. conducted a cross-sectional study on an existing cohort formed in 2002 from Houston, Texas [22]. Patients were originally recruited through local health department surveillance and routine screening of blood donations. The authors assessed 111 patients a median of 6.8 years post-infection (range: $0.1-11$ years), and concluded that patients with WNV-associated retinopathy had significantly lower SF-36 scores compared to patients without (mean: $93.2 \pm 14.8$ versus $100.2 \pm 8.3$ ). It is unclear which SF-36 health domain was being reported, and how a mean score was over the maximum of 100 . In the second study, Carson et al. recruited 49 patients from North Dakota in 2003 using state-based surveillance, and laboratory records [23]. SF-12 was assessed a mean of 13 months post-diagnosis (range: 10.5-15.8 months). Hospitalized patients had a mean Physical Component Summary score of $43.7 \pm 9.3$, and Mental Component Summary score of $51.6 \pm 11.5$. Nonhospitalized patients had statistically similar physical and mental scores ( $40.8 \pm 10.1$, and $47.8 \pm 10.2$, respectively). Comparisons to these two studies are difficult when the present work had different assessment time points, recruitment processes, catchment areas, patient sociodemographics, and numerical HRQoL scales.

There are several limitations to this study. Firstly, there was substantial attrition with $62 \%$ of patients lost to follow-up by one year. Specifically, $52 \%$ with neuroinvasive disease, and $68 \%$ with non-neuroinvasive were lost by then, introducing differential selection bias by oversampling the former subgroup. In the original cohort study, nine patients ended participation after having felt improvements to their health [5]. We may have underestimated HRQoL over the long-term if only the sickest are represented near the end of the study period. However, we may also have overestimated HRQoL due to survival bias where the healthiest patients remain alive through follow-up. We explored multiple imputation of missing data. Re-analysis using imputed datasets showed results similar to the analysis on only the observed data, suggesting robustness. Secondly, long-term HRQoL inferences were limited by a maximum of three years of follow-up. The first human case in Canada was reported in 2002 in Ontario [5]. Continual efforts to collect data can provide insights to neurological, functional, cognitive, and renal sequelae [26-29]. The longest follow-up in a North American cohort was ten years among patients identified through public health surveillance in 2002 in Houston, Texas [27]. While the WNV cohort from which our data originated was the largest in Canada, we only stratified patients by neuroinvasive and non-neuroinvasive diseases. Sample sizes did not allow for further stratification into encephalitis, meningitis, meningoencephalitis, and acute flaccid paralysis. An overall literature gap in syndrome-specific data remains, especially for patients with acute flaccid paralysis and meningoencephalitis [30].

\section{Conclusions}

Given the seasonal epidemics of WNV infections in North America, a greater understanding of HRQoL over the short and long term is warranted. Patients with WNV infection reported low HRQoL during acute illness, but demonstrated rapid improvements by six months of follow-up. Neuroinvasive disease status was not predictive of HRQoL over time. HRQoL accounts for patient preferences, providing important evidence for clinical, and health policy decision-making.

\section{Additional files}

Additional file 1: Table S1. Linear mixed-effects model for predictors of health-related quality of life (measured as untransformed utility scorest) over entire follow-up. (DOCX $15 \mathrm{~kb}$ )

Additional file 2: Table S2. Response feature analysis: Utility scores† summarized into mean of long-term utilities. Results are reported as means (95\% confidence interval). (DOCX $13 \mathrm{~kb}$ )

Additional file 3: Figure S1. Predicted values from best beta-regression model for area under the curve (AUC) past one year for patients with neuroinvasive disease (pink) and non-neuroinvasive disease (blue). (PDF $248 \mathrm{~kb}$ )

\section{Abbreviations}

HRQoL: health-related quality of life; HUI: Health Utilities Index; SF-

12: Medical Outcomes Survey Short Form 12; SF-36: Medical Outcomes Survey Short Form 36; SF-6D: Medical Outcomes Study Short Form 6D classification; WNV: West Nile virus

\section{Acknowledgements}

We thank the research nurses and coordinators of the West Nile longitudinal study for collecting and cleaning the data. We thank the study participants for generously giving their time. 


\section{Availability of data and materials}

The datasets used and/or analysed during the current study are available from the corresponding author on reasonable request.

\section{Authors' contributions}

Study design and/or data acquisition- all; Data analysis- MY, GT, BS; First draft- MY, BS; Subsequent drafts- all; Final approval- all.

\section{Ethics approval and consent to participate}

The present work is part of a larger study, Cost-effectiveness of West Nile virus intervention strategies. A computer simulation (Canadian Institutes of Health Research grant: MOP133571), approved by the Ontario Agency for Health Protection and Promotion institutional review board. Data were obtained from a previous study from McMaster University (MOP-69010), which had received ethics approval from McMaster University, University of Manitoba, University of Saskatchewan, and University of Alberta.

\section{Consent for publication}

Not applicable.

\section{Competing interests}

The authors declare that they have no competing interests.

\section{Publisher's Note}

Springer Nature remains neutral with regard to jurisdictional claims in published maps and institutional affiliations.

\section{Author details}

${ }^{1}$ Public Health Ontario, 480 University Avenue, Suite 300, Toronto, ON M5G 1V2, Canada. Institute of Health Policy, Management and Evaluation, University of Toronto, 155 College St, Toronto, ON M5T 3M6, Canada. ${ }^{3}$ Dalla Lana School of Public Health, University of Toronto, 155 College St, Toronto, ON M5T 3M6, Canada. ${ }^{4}$ University Health Network, 200 Elizabeth Street, Toronto, ON M5G 2C4, Canada. ${ }^{5}$ Department of Pathology and Molecular Medicine, McMaster University, 1280 Main Street West, Hamilton, ON L8S 4K1, Canada. ${ }^{6}$ Institute for Clinical Evaluative Sciences, 2075 Bayview Ave,

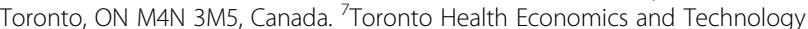
Assessment (THETA) Collaborative, 200 Elizabeth Street, Toronto, ON M5G 2C4, Canada.

\section{Received: 16 June 2017 Accepted: 11 October 2017}

\section{Published online: 23 October 2017}

\section{References}

1. Petersen LR, Brault AC, Nasci RS. West Nile virus: review of the literature. JAMA [Internet]. 2013;310(3):308-15. Available from: http://www. pubmedcentral.nih.gov/articlerender.fcgi?artid=4563989\&tool= pmcentrez\&rendertype=abstract. [cited 2015 Nov 2].

2. Drummond M, Sculpher M, Torrance G, O'Brien B, Stoddart G. Methods for the economic evaluation of health care programme. 3rd ed. Oxford: Oxford University Press; 2005.

3. Sejvar JJ, Curns AT, Welburg L, Jones JF, Lundgren LM, Capuron L, et al. Neurocognitive and functional outcomes in persons recovering from West Nile virus illness. J Neuropsychol [Internet]. 2008;2(Pt 2):477-99. Available from: http://www.ncbi.nlm.nih.gov/pubmed/19824176. [cited 2015 Nov 2].

4. Sadek JR, Pergam SA, Harrington JA, Echevarria LA, Davis LE, Goade D, et al. Persistent neuropsychological impairment associated with West Nile virus infection. J Clin Exp Neuropsychol [Internet]. 2010;32(1):81-7. Available from: http://www.ncbi.nlm.nih.gov/pubmed/19513920. [cited 2015 Nov 2].

5. Loeb M, Hanna S, Nicolle L, Eyles J, Elliott S, Rathbone M, et al. Prognosis after West Nile virus infection. Ann Intern Med. 2008;149(4):232-41.

6. Ware J, Snow K, Kosinsk iM, Gandek B, institute NEMCHH. SF-36 health survey: manual and interpretation guide: the health institute, New England Medical Center; 1993. Boston: The Health Institute, New England Medical Center; 1993.

7. Brazier J, Roberts J, Deverill M. The estimation of a preference-based measure of health from the SF-36. J Health Econ [Internet]. 2002;21(2):27192. Available from: http://www.ncbi.n/m.nih.gov/pubmed/11939242. [cited 2015 Sep 30].

8. von Neumann J, Morgenstern O. Theory of games and economic behavior. 3rd ed. New York: Wiley; 1953.
9. Brazier JE, Roberts J. The estimation of a preference-based measure of health from the SF-12. Med Care [Internet]. 2004;42(9):851-9. Available from: http://www.ncbi.nlm.nih.gov/pubmed/15319610. [cited 2015 Sep 30].

10. Core Team R. R: a language and environment for statistical computing. Vienna: R foundation for statistical computing. URL https://www.R-project. org/. Available from: 2016. https://www.r-project.org.

11. Walters S. Quality of life outcomes in clinical trials and health-care evaluation: a practical guide to analysis and interpretation: Wiley; 2009.

12. Matthews JN, Altman DG, Campbell MJ, Royston P. Analysis of serial measurements in medical research. BMJ [Internet]. 1990;300(6719):230-5. Available from: http://www.pubmedcentral.nih.gov/articlerender.fcgi?artid= 1662068\&tool=pmcentrez\&rendertype=abstract. [cited 2015 Nov 2].

13. Smithson M, Verkuilen J. A better lemon squeezer? Maximum-likelihood regression with beta-distributed dependent variables. Psychol Methods [Internet]. 2006;11(1):54-71. Available from: http://www.ncbi.nlm.nih.gov/ pubmed/16594767. [cited 2015 Aug 27].

14. Cribari-Neto F, Zeileis A. Beta regression in R. J Stat Softw. 2010:34(2):1-24. URL http://www.jstatsoft.org/v34/i02/.

15. Rubin D. Multiple imputation for nonresponse in surveys. New York: Wiley; 1987.

16. Klee AL, Maidin B, Edwin B, Poshni I, Mostashari F, Fine A, et al. Long-term prognosis for clinical West Nile virus infection. Emerg Infect Dis [Internet]. 2004;10(8):1405-11. Available from: http://www.pubmedcentral.nih.gov/ articlerender.fcgi?artid=3320418\&tool=pmcentrez\&rendertype=abstract. [cited 2015 Aug 7].

17. Mittmann N, Trakas K, Risebrough N, Liu BA. Utility scores for chronic conditions in a community-dwelling population. Pharmacoeconomics [Internet]. 1999;15(4):369-76. Available from: http://www.ncbi.nlm.nih.gov/ pubmed/10537955. [cited 2014 Apr 22].

18. Feeny D, Furlong W, Torrance GW, Goldsmith CH, Zhu Z, DePauw S, et al Multiattribute and single-attribute utility functions for the health utilities index mark 3 system. Med Care [Internet]. 2002:40(2):113-28. Available from: http://www.ncbi.n/m.nih.gov/pubmed/11802084. [cited 2016 Aug 12]

19. Custer B, Busch MP, Marfin AA, Petersen LR. The cost-effectiveness of screening the U.S. blood supply for West Nile virus. Ann Intern Med [Internet]. 2005;143(7):486-92. Available from: http://www.ncbi.nlm.nih.gov/ pubmed/16204161. [cited 2016 Aug 11].

20. Korves CT, Goldie SJ, Murray MB. Cost-effectiveness of alternative bloodscreening strategies for West Nile Virus in the United States. PLoS Med [Internet]. 2006;3(2):e21. Available from: http://www.pubmedcentral.nih.gov/ articlerender.fcgi?artid=1324950\&tool=pmcentrez\&rendertype=abstract. [cited 2015 Oct 13].

21. Samaan Z, McDermid Vaz S, Bawor M, Potter TH, Eskandarian S, Loeb M, et al. Neuropsychological Impact of West Nile Virus Infection: An Extensive Neuropsychiatric Assessment of 49 Cases in Canada. Klein RS, editor. PLoS One [Internet]. Public Library of Science; 2016;11(6):e0158364. Available from: http://dx.plos.org/10.1371/journal.pone.0158364. [cited 2016 Aug 10]

22. Hasbun R, Garcia MN, Kellaway J, Baker L, Salazar L, Woods SP, et al. West Nile Virus Retinopathy and Associations with Long Term Neurological and Neurocognitive Sequelae. PLoS One [Internet]. 2016;11(3):e0148898. Available from: http://www.ncbi.nlm.nih.gov/pubmed/26950214. [cited 2016 Aug 11].

23. Carson PJ, Konewko P, Wold KS, Mariani P, Goli S, Bergloff P, et al. Longterm clinical and neuropsychological outcomes of West Nile virus infection. Clin Infect Dis [Internet]. 2006;43(6):723-30. Available from: http://www.ncbi. nlm.nih.gov/pubmed/16912946. [cited 2016 Aug 11].

24. Petersen LR, Marfin AA, Gubler DJ. West Nile virus. JAMA [Internet]. 2003; 290(4):524-8. Available from: http://www.ncbi.nlm.nih.gov/pubmed/ 12876096. [cited 2015 Aug 7].

25. Couturier E, Guillemin F, Mura M, Léon L, Virion J-M, Letort M-J, et al. Impaired quality of life after chikungunya virus infection: a 2-year follow-up study. Rheumatology (Oxford) [Internet]. Oxford University Press; 2012;51(7): 1315-22. Available from: http://www.ncbi.nlm.nih.gov/pubmed/22427407. [cited 2016 Aug 11].

26. Patel H, Sander B, Nelder MP. Long-term sequelae of West Nile virus-related illness: a systematic review. Lancet Infect Dis [Internet]. 2015;15(8):951-9. Available from: http://www.ncbi.nlm.nih.gov/pubmed/26163373. [cited 2016 Feb 18].

27. Weatherhead JE, Miller VE, Garcia MN, Hasbun R, Salazar L, Dimachkie MM, et al. Long-term neurological outcomes in West Nile virus-infected patients: an observational study. Am J Trop Med Hyg [Internet]. 2015;92(5):1006-12. 
Available from: http://www.pubmedcentral.nih.gov/articlerender.fcgi?artid= 4426557\&tool=pmcentrez\&rendertype=abstract. [cited 2015 Aug 7].

28. Sejvar JJ. The long-term outcomes of human West Nile virus infection. Clin Infect Dis [Internet]. 2007:44(12):1617-24. Available from: http://www.ncbi. nlm.nih.gov/pubmed/17516407. [cited 2015 Aug 11].

29. Nolan MS, Podoll AS, Hause AM, Akers KM, Finkel KW, Murray KO.

Prevalence of chronic kidney disease and progression of disease over time among patients enrolled in the Houston West Nile virus cohort. PLoS One [Internet]. 2012;7(7):e40374. Available from: http://www.ncbi.nlm.nih.gov/ pubmed/22792293. [cited 2016 Aug 12].

30. Yeung MW, Shing E, Nelder M, Sander B. Epidemiologic and clinical parameters of West Nile virus infections in humans: a scoping review. BMC Infect Dis [Internet]. 2017;17(1):609. Available from: https://doi.org/10.1186/ s12879-017-2637-9.

Submit your next manuscript to BioMed Central and we will help you at every step:

- We accept pre-submission inquiries

- Our selector tool helps you to find the most relevant journal

- We provide round the clock customer support

- Convenient online submission

- Thorough peer review

- Inclusion in PubMed and all major indexing services

- Maximum visibility for your research

Submit your manuscript at www.biomedcentral.com/submit 